Revue internationale P.M.E.

Économie et gestion de la petite et moyenne entreprise

\title{
Transmissions internes et transmissions externes dans les PME françaises : existe-t-il des différences de changements stratégiques et d'orientations stratégiques?
}

\section{Slimane Haddadj et Aude D'Andria}

Volume 11, numéro 4, 1998

URI : https://id.erudit.org/iderudit/1009051ar

DOI : https://doi.org/10.7202/1009051ar

Aller au sommaire du numéro

Éditeur(s)

Presses de l’Université du Québec

ISSN

0776-5436 (imprimé)

1918-9699 (numérique)

Découvrir la revue

Citer cet article

Haddadj, S. \& D'Andria, A. (1998). Transmissions internes et transmissions externes dans les PME françaises : existe-t-il des différences de changements stratégiques et d'orientations stratégiques ? Revue internationale P.M.E., 11(4), 45-65. https://doi.org/10.7202/1009051ar
Résumé de l'article

À partir d'une enquête menée auprès d'un échantillon de 83 entreprises, cette recherche montre que les changements stratégiques des entreprises qui mettent en place des transmissions internes ne diffèrent pas de celles qui mettent en place des transmissions externes2. Par ailleurs, elle cherche à savoir en quoi les orientations stratégiques des entreprises qui suivent la transmission peuvent être expliquées par la perception que se font les dirigeants de l'environnement. 


\title{
Transmissions internes et transmissions externes dans les PME françaises: existe-t-il des différences de changements stratégiques et d'orientations stratégiques?
}

\author{
Slimane HADDADJ \\ Institut d'administration des entreprises de Lille \\ Aude D'ANDRIA \\ Institut universitaire de technologie de Brétigny
}

\section{MOTS CLÉS}

\section{Changement de dirigeant - Contingence environnementale Entreprises familiales - Orientations stratégiques Transmission d'entreprise}

\begin{abstract}
RÉSUMÉ
À partir d'une enquête menée auprès d'un échantillon de 83 entreprises, cette recherche montre que les changements stratégiques des entreprises qui mettent en place des transmissions internes ne diffèrent pas de celles qui mettent en place
\end{abstract}

\section{LES AUTEURS}

Après un séjour de plus d'un an et demi aux États-Unis, Slimane Haddadj intègre l'Institut d'administration des entreprises de Lille en tant que maître de conférences. Au sein du Graphe, dirigé par le professeur Pierre Louart, il collabore avec des chercheurs d'Amérique du Nord et travaille particulièrement sur les stratégies des entreprises dans le cadre des transmissions d'entreprise ${ }^{1}$. Adresse: IAE de Lille, 104, avenue du Peuple-Belge, 59000 Lille (France). Téléphone: 33.3.20.12.34.85.

Détentrice d'un doctorat en sciences de gestion à l'IAE de Lille, Aude D'Andria est maître de conférences à l'Institut universitaire de technologie de Brétigny. En 1996, elle a décidé de se familiariser avec le contexte des PME et entamé des recherches sur ce sujet. Adresse: IUT d'Evry-Brétigny, Château La Fontaine, 91731 Brétigny s/Orge, France. Téléphone: 33.1.46.61.19.87.

1. Je tiens à remercier la Fondation nationale pour l'enseignement de la gestion des entreprises pour son aide financière à ce projet. Sans elle, cet article n'aurait jamais pu être réalisé. Par ailleurs, je tiens à remercier les professeurs A. Bernard (Essec), R.A. Thiétart (Université Paris-Dauphine) et Pierre Louart (Université de Toulouse) de leur soutien constant. 
des transmissions externes². Par ailleurs, elle cherche à savoir en quoi les orientations stratégiques des entreprises qui suivent la transmission peuvent être expliquées par la perception que se font les dirigeants de l'environnement.

\begin{abstract}
This paper explores the connection between CEO transmission and strategic considerations in small and medium-sized firms. Specifically, it examines whether external transmission differs from internal transmission in its strategic change, and whether the firm's strategic patterns can be explained by the CEO's perception of the business environment.
\end{abstract}

\title{
RESUMEN
}

Este artículo examina las características de las estrategias de la PyME. El autor considera que la sucesión del dirigente (interno o externo) acarrea diferentes estrategias. Luego sigue una discusión de la manera en que la percepción que tiene un dirigente de todo lo que pertenece al medio ambiente influye sus estrategias.

\section{Problématique}

De tous les événements récurrents que connaît l'entreprise de petite et moyenne taille de type familial, celui du transfert du pouvoir et de l'autorité lors de la transmission est le plus critique pour l'organisation (Handler, 1994). Point de rupture obligée entre un dirigeant et un repreneur, la transmission de l'entreprise conditionne l'avenir et la pérennité de celle-ci (Garach et Ganitsky, 1995). Or, les dirigeants qui partent à la retraite ne parviennent pas tous à trouver de repreneur : en France, 2000 entreprises disparaissent sans trouver de repreneur et 2000 autres déposent leur bilan en raison d'un problème de transmission (Lehmann, 1993). Mais ces chiffres sont loin d'être expliqués par un déterminisme absolu puisque la transmission de l'entreprise découle de la volonté du dirigeant lui-même (Hirigoyen, 1987) et celui-ci dispose de plusieurs moyens d'action pour assurer son départ lorsqu'il en a l'occasion (Lansberg et Astrachan, 1994). Il peut soit transmettre son entreprise à l'un (ou plusieurs) de ses enfants (ou membres de sa famille), soit transmettre à des personnes extérieures au clan familial. Dans le premier cas, la conservation patrimoniale de l'entreprise exige que les héritiers s'y engagent et qu'ils soient en mesure de la gérer pour éviter que celle-ci ne disparaisse (Garach et Ganitsky, 1995).

2. Par transmission interne, il est entendu que ce sont les salariés de l'entreprise qui rachètent l'entreprise et qui en possèdent au moins la majorité du capital. Ce cas de figure est opposé à la transmission externe où ce sont des personnes extérieures (personnes physiques ou entreprises) qui rachètent l'entreprise. 
Dans le deuxième cas, il s'agit ni plus ni moins d'une dissolution du patrimoine familial (Barcelo, 1988) ${ }^{3}$.

Puisque plusieurs options s'offrent au dirigeant en matière de transmission et puisque la rupture dans la chaîne de la continuité familiale au profit de personnes extérieures est de plus en plus fréquente, s'interroger sur les changements et les orientations stratégiques qui accompagnent la transmission devient légitime. Dès lors, surgissent un certain nombre de questions. Comment le développement stratégique est-il assuré à travers le changement de dirigeant? En quoi les repreneurs externes agissent-ils différemment des repreneurs internes ? Comment la perception de l'environnement peut-elle influer sur les choix stratégiques des entreprises?

C'est pourquoi, dans un premier temps, cette recherche va montrer si les PME de type familial qui font appel à des repreneurs internes, comparées à celles qui font appel à des repreneurs externes, introduisent des changements stratégiques différents (mesurés à partir des conséquences stratégiques). Puis, dans un second temps, nous vérifierons si la diversité des orientations stratégiques des entreprises (toujours mesurées à partir des conséquences stratégiques), mises en place par les nouveaux dirigeants, dépend des perceptions du contexte environnemental dans lequel elles évoluent.

\section{Fondements théoriques}

\section{Le rôle du dirigeant dans le développement de l'entreprise}

En se référant particulièrement à l'analogie biologique, la théorie du cycle de vie propose une liaison possible entre l'évolution de l'entreprise et l'évolution du dirigeant (Dodge, Fullerton et Robbins, 1994). Bien que divergeant quant au nombre de stades qui forment le cycle de vie de l'entreprise, les auteurs convergent sur le principe que toutes les entreprises suivent le même sentier d'évolution (Julien, 1994). Selon eux, toute organisation doit passer par des stades d'évolution distincts, mais prévisibles, qui se suivent selon une suite logique et qui ne sont pas réversibles (Dodge et al. 1994). Tantôt normatifs, tantôt descriptifs des différentes phases de

3. À cet égard, la loi du 9 juillet 1984 ajoute un trait supplémentaire aux logiques des reprises par des personnes extérieures à la famille puisqu'elle donne la possibilité aux salariés, par des allégements des charges fiscales et en facilitant les sources d'emprunts, de pouvoir racheter l'entreprise dans laquelle ils travaillent. Cette loi a donc permis de créer un nouvel artifice en matière de transmission, puisque les salariés repreneurs, jusque-là exceptionnels, peuvent dorénavant entrer sur la scène successorale. Les chiffres relatent assez bien le succès de cette nouvelle « concurrence » entre repreneurs extérieurs puisque, dans $45 \%$ des cas de transmissions, les repreneurs sont des salariés de l'entreprise, dans $39 \%$ des cas, des personnes physiques extérieures et, dans $16 \%$ des cas, une autre entreprise (CEPME, 1990). 
développement de l'entreprise, ces modèles adoptent de manière assez explicite une attitude orientée vers la proactivité. Par exemple, Greiner (1972) et Churchill et Hatten (1987) adoptent une perspective selon laquelle l'évolution des entreprises nécessite un style de management particulier à chaque stade de développement, remettant ainsi fortement en cause la légitimité du dirigeant à faire évoluer son entreprise. La personnalité du dirigeant peut même devenir un obstacle à l'évolution de l'entreprise, notamment lorsque celui-ci voit à travers elle la continuité de luimême, ou bien lorsqu'il se révèle incapable de faire la différence entre l'avenir de la «progéniture » de son propre parcours professionnel (Kets de Vries, 1985). Au-delà des styles de management variables selon le contexte, il est tout à fait possible de préconiser le départ du dirigeant pour ne pas remettre en cause la pérennité et le développement de l'entreprise (Weinshall et Vickery, 1987). Dans ce cas, il doit être remplacé de préférence par une nouvelle équipe de direction qui correspond mieux au nouveau contexte organisationnel et qui pourra mettre en place les changements nécessaires à la survie de l'entreprise. Toutefois, si le changement de dirigeant est souvent proposé, le départ effectif n'est jamais sans risque. Tout dépend du stade de développement atteint par l'entreprise. Par exemple, une transmission dans une entreprise mature s'avère moins risquée, car le développement de l'entreprise n'est pas nécessairement lié à la présence du dirigeant et l'entreprise a certainement élaboré une procédure lui permettant de favoriser le changement de dirigeant.

Fortement inspirée d'une idéologie de croissance dans laquelle le dirigeant est plus souvent assimilé à un entrepreneur au centre du développement de l'entreprise, l'approche proposée par la théorie du cycle de vie doit être nuancée dans la mesure où l'environnement y est totalement ignoré (Dodge et al. 1994). Il est nécessaire de tenir compte de la nature du jeu concurrentiel qui, nourri par les forces du marché et les pressions concurrentielles, explique le développement de l'entreprise. Par conséquent, le caractère séquentiel et linéaire des modèles faisant référence au cycle de vie reste peu compatible avec la diversité des orientations stratégiques mises en place lors du changement de dirigeant dans le cadre des transmissions d'entreprise (Dodge et al. 1994). Dans ce sens, l'étude réalisée sur un échantillon d'entreprises à haute technologie est significative (Willard et Krueger, 1992). Les auteurs ont montré que les dirigeants fondateurs des entreprises ont su s'adapter à la complexité croissante induite par la croissance rapide sans toutefois mettre en péril la performance de l'entreprise, ni même en perdre le contrôle, remettant ainsi fortement en cause la théorie du cycle de vie.

Même si les recherches restent controversées pour savoir si véritablement le développement de l'entreprise s'explique par l'évolution du dirigeant ou si, au contraire, le développement de l'entreprise est tributaire du contexte environnemental, il reste que, chaque année, 50000 entreprises changent de mains (CEPME, 1990; INSEE, 1997). À partir de là, la piste de la compréhension de la transmission, suivant la nature des repreneurs, devient pertinente. Ainsi, il a été 
constaté, par exemple, que le créateur d'une entreprise était plus « entrepreneurial » que le dirigeant qui en héritait dans le cadre familial ou qui la rachetait au dirigeant précédent (Cooper et Dunkelberg, 1986). Par ailleurs, un dirigeant qui crée son entreprise serait plus souvent à la recherche d'occasions d'affaires et d'activités très risquées, mais aussi très rentables (Stevenson, Roberts et Grousbeck, 1985). La raison avancée serait que le dirigeant qui hérite de l'entreprise doit gérer un risque potentiel de conflit interne lié à la reprise de l'entreprise (Ward, 1987). Du même coup, il peut ne pas bénéficier de la coopération et de la confiance de l'équipe managériale en poste. Quant au repreneur extérieur, il peut être placé dans une situation où, ne connaissant pas encore toute la complexité des activités et des marchés de l'entreprise, il peut difficilement œuvrer efficacement pour son développement (Niemann, 1991).

Une lecture rapide de ces cas de figure pourrait conduire à considérer que les entreprises qui sont créées réalisent de meilleures performances que celles qui sont reprises. Or, des cas de figure inverses sont possibles. Par exemple, il a été montré que les entreprises reprises par des membres de la famille ou par des repreneurs extérieurs pouvaient plus facilement assurer leur développement que celles qui se créent, car l'accès aux compétences managériales et aux ressources financières leur étaient plus aisées (Brokaw, 1992). De même, les entreprises qui se créent peuvent éprouver, au cours de leur développement, des difficultés de délégation dans la mesure où le dirigeant est encore trop identifié à son entreprise (Buchele, 1965).

Bien que enrichissantes, ces considérations restent encore trop descriptives. Aussi n'aident-elles pas réellement à comprendre le lien qui peut exister entre la nature du dirigeant (créateur ou repreneur) et la performance de l'entreprise. Dans la mesure où les différents styles entrepreneuriaux pratiqués dans une entreprise ne permettent pas de rendre compte des niveaux de performance des entreprises, Chaganti et Schnerr (1994), en reprenant la classification de Cooper et al. (1986), ont montré que le créateur d'une entreprise réalisait de meilleures performances en termes de rentabilité que celui qui en héritait dans le cadre familial ou qui la rachetait. Ces différences sont attribuées non seulement au principe selon lequel le créateur d'entreprise est installé dans une logique de circonstances opportunes, alors que le repreneur ou l'héritier ne l'est pas, mais aussi au fait que les pratiques managériales sont déterminées par les choix stratégiques des dirigeants.

\section{Actions du dirigeant sur l'environnement}

Selon Stoner (1982), nombreuses sont les entreprises familiales qui estiment que la prise en compte de l'environnement dans les décisions stratégiques n'est pas importante. A contrario, d'autres travaux ont souligné l'importance de considérer l'environnement dans l'élaboration de la stratégie des PME (Sexton et Van Auken, 1982 ; Daft, Sormunen et Parks, 1988). Plus précisément, la différence entre des entreprises ayant obtenu des performances faibles et celles ayant obtenu des 
performances élevées s'expliquerait par le fait que seules les entreprises ayant une vision large de l'environnement et qui l'analysent souvent ont de meilleures performances que celles qui ne le font pas ou peu souvent (Daft et al., 1988). En outre, si l'une des tâches qui incombent au dirigeant est bien d'analyser et de comprendre l'environnement afin de donner une position confortable à son entreprise, « les informations dont dispose le dirigeant devront être triées, rassemblées, mises en forme de manière à ce que le responsable puisse prendre les mesures nécessaires à une riposte rapide face à une menace ou puisse anticiper une possibilité présente sur le marché » (Thiétart, 1987). En d'autres termes, pour appliquer une stratégie qui réponde à l'exigence de l'environnement, l'entreprise doit avoir une compréhension complète des forces qui animent cet environnement. Parmi ces forces, l'entreprise tiendra compte des concurrents qui sont positionnés sur son marché et également des fournisseurs, des clients, des produits de substitution, des nouveaux entrants et des entrants potentiels (Porter, 1985).

Si le fait de prendre en considération l'environnement dans les décisions stratégiques pour améliorer la performance de l'entreprise a donné lieu à de nombreuses controverses (Robinson et Pearce, 1984 ; Orpen, 1994), le débat porte aussi sur la nature de l'environnement. D'un côté, les tenants de l'école de l'écologie des populations affirment que l'environnement est une donnée objective et que cet environnement est le principal déterminant des décisions stratégiques (Aldrich, 1979; Greenfield et Strickon, 1981). Par exemple, on a avancé que les pressions environnementales condamnaient à faire disparaître toutes les entreprises qui ne se soumettaient pas suffisamment aux impératifs de l'environnement (Greenfield et Strickon, 1981). L'environnement sélectionnerait ainsi les entreprises sans que celles-ci ne puissent véritablement influer sur leurs chances de succès. De l'autre côté, les tenants du choix stratégique considèrent que l'environnement relève d'un état interprétatif du dirigeant (Child, 1972; Gartner, 1985). Bien que cette controverse subsiste, il est de plus en plus reconnu que l'environnement ne serait pas une donnée, mais plutôt un construit, une réalité perçue par les acteurs (Weick, 1969). Les dirigeants d'entreprise décrètent et mettent en scène l'environnement auquel leur entreprise doit s'adapter à partir des représentations mentales qu'ils se font de la réalité qui les entoure (Silvestre et Goujet, 1996). Ils déterminent alors leurs choix stratégiques afin d'atteindre une meilleure congruence entre l'entreprise et son environnement. Par conséquent, le rôle du dirigeant dans les décisions stratégiques est important et, a fortiori, le rôle interprétatif du dirigeant dans le choix des décisions stratégiques joue sur le devenir de l'entreprise.

\section{Hypothèses}

Les recherches menées par Robinson et Pearce (1984) et Dodgson et Rothwell (1989) ont montré que les décisions stratégiques des entreprises et le management des choix stratégiques sont des préoccupations qui touchent les PME, puisque leur 
développement et leur survie en dépendent. De surcroît, construire une théorie spécifique à la PME est nécessaire ; mais la construire à partir d'études empiriques conceptualisées dans le champ spécifique de la PME pour des raisons de validité devient primordial (Robinson et Pearce, 1984). Bien que certaines recherches se soient orientées vers des aspects assez réducteurs de la réalité organisationnelle, la reliant directement à la performance de l'organisation (D'Amboise et Muldowney, 1988) ou, encore, n'ayant adopté qu'une perspective unidimensionnelle (Gartner, 1985), la considération de dimensions holistes est indispensable pour les recherches à venir. Ces dimensions couvriraient à la fois l'environnement de l'entreprise, les configurations organisationnelles et les caractéristiques managériales (Merz et Sauber, 1995). C'est pourquoi notre recherche repose sur le postulat que la compréhension liant le dirigeant au développement de l'entreprise ne peut se faire qu'en intégrant à la fois l'origine de la transmission, les stratégies adoptées par les nouveaux dirigeants et les caractéristiques de l'organisation et de l'environnement. C'est donc dans le cadre de ce postulat que notre échantillon a été construit afin de pouvoir s'interroger sur les changements et les orientations stratégiques qui caractérisent les transmissions internes et les transmissions externes dans le cadre d'entreprises qui perdent leur statut d'entreprise familiale.

Hypothèse 1: Les changements stratégiques apportés par les repreneurs internes sont différents de ceux apportés par les repreneurs externes.

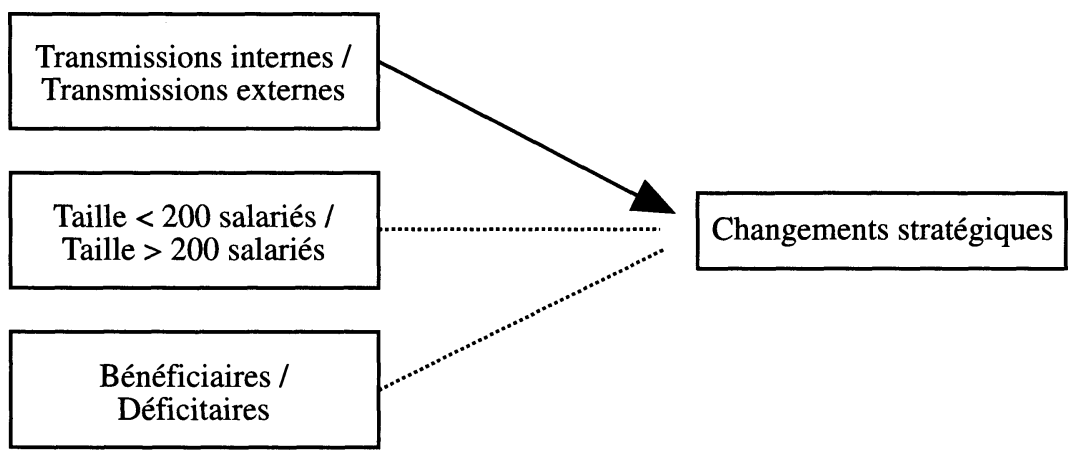

Les lignes pleines représentent l'hypothèse et les lignes en pointillé, les variables de contrôle.

Hypothèse 2: Les comportements stratégiques adoptés par les repreneurs internes et externes sont fonction de la perception qu'ont les repreneurs de leur environnement. 


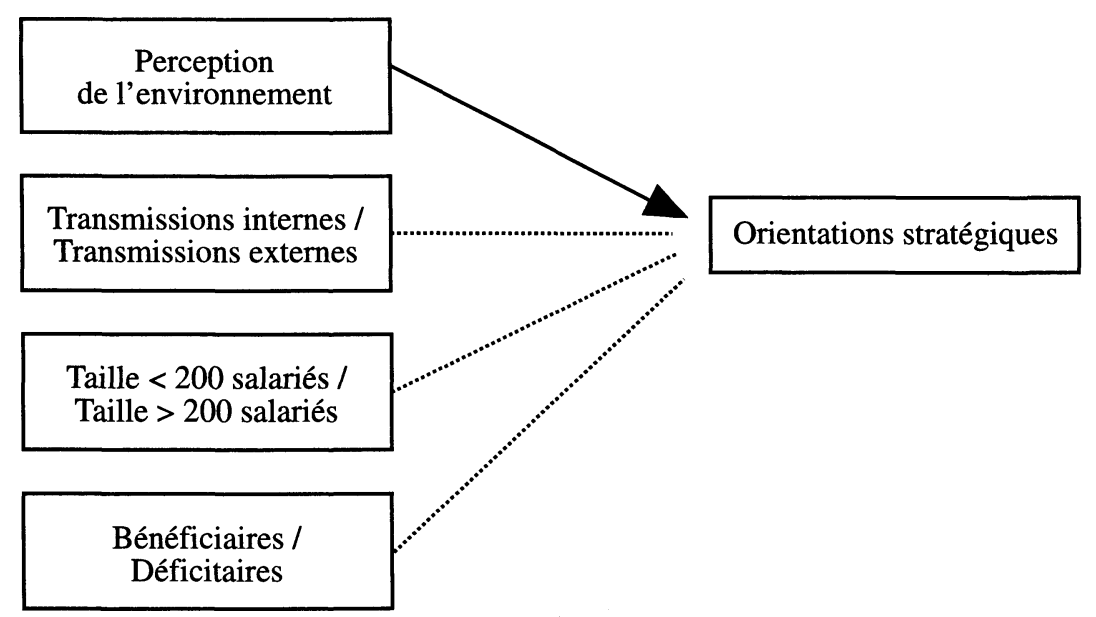

Les lignes pleines représentent l'hypothèse et les lignes en pointillé, les variables de contrôle.

\section{Méthodologie de recherche}

\section{Caractéristiques de l'échantillon}

Bien que quelques cas de reprise d'entreprise, principalement de grandes entreprises, soient régulièrement relevés dans la presse, aucune base de données officielle, aisément accessible, ne semble exister en France. Notre démarche a donc consisté à contacter, dans un premier temps, tous les Syndicats de développement régional (SDR) et toutes les Chambres de commerce et de l'industrie (CCI). Nous leur avons demandé de nous indiquer les cas de transmission d'entreprises dont ils étaient informés dans leur région respective. Un échantillon initial de 157 entreprises a pu être constitué. Dans un deuxième temps, nous avons, durant le printemps 1990, pris contact par téléphone avec toutes les entreprises mentionnées. À la suite de ces différents entretiens, nous avons retenu un échantillon de 117 entreprises en appliquant les deux critères suivants :

- l'effectif ne devait pas dépasser 500 salariés $^{4}$;

- l'entreprise devait avoir connu une transmission depuis au moins deux ans ${ }^{5}$.

Malgré le contrôle initial effectué lors des entretiens téléphoniques, il est apparu lors du retour du questionnaire, durant l'été et l'automne 1990, que 12 entreprises

4. Caractéristique de la PME en France. Pour plus de précisions, voir Julien (1994).

5. Il fallait laisser aux repreneurs un laps de temps de deux ans nécessaire pour mesurer les réalisations stratégiques. 
dépassaient le seuil des 500 salariés. Elles ont donc été retirées de l'échantillon afin de respecter le premier critère (effectif inférieur ou égal à 500 salariés). En ce qui concerne le second critère (avoir connu une transmission depuis au moins deux ans), il est apparu, lors du retour du questionnaire, que toutes les entreprises étudiées avaient connu une transmission depuis au plus cinq ans (et au moins deux ans).

Le taux de retour global des questionnaires est de $86 \%$ et le taux de réponses exploitables, de $75 \%$. Ces taux élevés s'expliquent par le contact téléphonique établi avec les dirigeants ou avec l'un de leurs proches collaborateurs avant l'envoi du questionnaire.

Les entreprises de l'échantillon appartiennent au secteur privé, ne sont pas cotées en Bourse, n'ont pas de filiale à l'étranger, même si certaines d'entre elles sont engagées dans des activités d'exportation de leurs produits. Les entreprises étudiées ont un effectif variant de six à 490 salariés, avec une moyenne de 129 salariés et un écart type de 121. Plusieurs secteurs sont représentés : 53 entreprises appartiennent au secteur de l'industrie, 19, à celui des services, neuf, à celui du bâtiment et travaux publics, et deux, à celui du transport.

L'origine des repreneurs a été différenciée selon la classification du CEPME (1990) pour lequel la transmission interne est définie comme le rachat de l'entreprise par les individus en poste dans l'entreprise, alors que la transmission externe désigne le rachat par les personnes physiques ou morales qui ne sont pas en poste dans l'entreprise. Avec cette distinction, l'échantillon final se compose de 53 entreprises ayant connu une transmission externe et de 30 entreprises ayant connu une transmission interne. Concernant les transmissions internes, il est apparu que la transmission s'est faite au profit de plusieurs salariés ; quant aux transmissions externes, seules des personnes morales ont repris les entreprises. Avant que la transmission ne se réalise, la majorité du capital de l'entreprise était directement détenue par le dirigeant ou par sa famille qui, en exerçant une influence importante sur la gestion de l'entreprise, faisait de l'entreprise une entreprise familiale (Perreault, 1994).

\section{Choix des variables stratégiques}

Les variables choisies l'ont été pour leur pertinence avec les théories existantes en matière de stratégie dans le cadre des PME, et aussi en fonction de la disponibilité des informations dans les entreprises étudiées. Elles ont permis de mesurer la variation des changements et des orientations stratégiques, variation mesurée à partir des conséquences stratégiques (entre la période qui précède la transmission et la période qui la suit). Il s'agit de la variation du taux de croissance du chiffre d'affaires, du nombre de domaines d'activité de l'entreprise, du nombre de clients, du chiffre d'affaires réalisé avec les anciens clients, du nombre de produits, de la qualité des produits et des services et des prix des produits. 
1. Le taux de croissance du chiffre d'affaires a été mesuré en comparant le taux moyen de croissance du chiffre d'affaires de l'entreprise des trois années précédant la transmission et le taux moyen de croissance du chiffre d'affaires de l'entreprise après la transmission.

2. Les domaines d'activité de l'entreprise ont été mesurés en comparant la moyenne du nombre de domaines d'activité de l'entreprise des trois années précédant la transmission et la moyenne du nombre de domaines d'activité de l'entreprise après la transmission.

3. Le nombre de clients a été mesuré en comparant la moyenne du nombre de clients des trois années précédant la transmission et la moyenne du nombre de clients après la transmission.

4. Le chiffre d'affaires réalisé avec les anciens clients a été mesuré en comparant le taux moyen du chiffre d'affaires réalisé avec les anciens clients de l'entreprise au cours des trois années précédant la transmission et le taux moyen du chiffre d'affaires réalisé avec les anciens clients après la transmission.

5. Le nombre de produits a été mesuré en comparant la moyenne du nombre de produits des trois années précédant la transmission et la moyenne du nombre de produits après la transmission.

6. La qualité des produits sur l'ensemble des produits de l'entreprise a été mesurée en comparant la qualité des produits des trois années précédant la transmission et la qualité des produits après la transmission ${ }^{6}$.

7. La qualité des services rendus sur l'ensemble des activités de l'entreprise a été mesurée en comparant la qualité des services rendus avant (trois années avant la transmission) et après la transmission (les années qui suivent la transmission $)^{7}$.

8. Les prix des produits (sur l'ensemble des produits et des services) ont été mesurés en comparant la moyenne des prix des trois années précédant la transmission et la moyenne des prix après la transmission.

\section{Mesure des variables stratégiques}

La mesure des variables stratégiques utilisées dans le questionnaire a été réalisée sur une échelle à trois dimensions : « inférieur », «égal », « supérieur ». Dans la

6. Dans l'énoncé, il était indiqué que la comparaison devait se faire par rapport aux éléments concernant directement la qualité et la fiabilité des produits.

7. Dans l'énoncé, il était indiqué que la comparaison devait se faire par rapport aux éléments concernant les délais de livraison et les retards dans la livraison des produits. 
mesure où nous nous intéressons aux variations stratégiques adoptées depuis la transmission du dirigeant, toutes les questions avaient pour but de déterminer la variation des changements apportés par les nouveaux dirigeants (mesurés à partir des conséquences stratégiques) depuis la transmission.

\section{Variables de contrôle}

À côté des variables mesurant les aspects stratégiques des entreprises, des variables de contrôle ont été établies afin de mesurer l'impact de la taille de l'entreprise ainsi que de la santé financière de l'entreprise avant et après la transmission sur les changements stratégiques et les orientations stratégiques.

Concernant la taille des entreprises ${ }^{8}$, les entreprises dont l'effectif était inférieur à 200 salariés ont été codées « zéro » et celles dont l'effectif était compris entre 200 et 500 salariés ont été codées «un».

Concernant la situation financière, il s'agissait de différencier les entreprises qui étaient bénéficiaires de celles qui étaient déficitaires au moment de la transmission. Pour cela, les entreprises déficitaires (dans l'année précédant la transmission) ont été codées «zéro », et celles qui ont été bénéficiaires (dans l'année précédant la transmission) ont été codées «un».

\section{Variables de contingence environnementale}

Pour compléter les questions fermées, il a également été demandé aux dirigeants interrogés de justifier et de préciser chacune de leurs réponses. Ce faisant, il a été possible de compléter les questions portant sur les variables stratégiques par des informations décrivant l'environnement dans lequel évoluent les entreprises. Ainsi, comme le souligne Jick (1979), les méthodes qualitatives peuvent jouer un rôle important en élucidant les données et peuvent aider à établir des conclusions auxquelles ne pourraient pas conduire d'autres méthodes. Dès lors, les questions ouvertes complètent les questions fermées en permettant de mieux interpréter les résultats. Il a donc été possible de mieux contrôler les facteurs contingents externes dans lesquels les entreprises se positionnent et évoluent, et d'analyser la façon dont les nouveaux dirigeants perçoivent leur environnement depuis que la transmission a eu lieu.

8. Comme le faisait remarquer un évaluateur, la dichotomie gagnerait à être scindée différemment pour reprendre la classification habituelle de la PME (< à 50 salariés, entre 50 et 200 salariés, > à 200 salariés). Après avoir effectué ce travail, il ne semble pas y avoir de différence entre la classification proposée et la nôtre. En conséquence, nous gardons notre classification. 


\section{Analyses statistiques}

Plusieurs analyses statistiques ont été effectuées sur les données de l'échantillon. Pour répondre à la première hypothèse, une analyse multivariée a été entreprise (MANOVA), complétée par des analyses univariées (ANOVA). Pour vérifier la seconde hypothèse, une analyse typologique à travers une analyse hiérarchique a été réalisée. Les huit variables mesurant les orientations stratégiques ont été utilisées comme les variables permettant de faire l'analyse typologique. Une seule rotation a été nécessaire. De plus, comme il n'existe pas de méthode objective permettant de déterminer le nombre exact de groupes (Hambrick, 1984), mais qu'il faut néanmoins assurer une objectivité dans le choix du nombre de groupes, une analyse de variance sur la moyenne de chaque variable a été pratiquée. Ainsi, il a été possible d'établir l'existence de cinq groupes. Enfin, pour vérifier si les groupes dégagés s'établissent de manière aléatoire, des analyses du khi carré ont été effectuées en fonction de l'origine de la transmission, de la taille de l'entreprise et de la situation financière avant la transmission.

\section{Présentation des résultats}

Les statistiques descriptives (coefficients de corrélation des variables, moyenne et écart type) sont présentées dans le tableau 1.

\section{TABleau 1}

Coefficient de corrélation de Spearman, moyenne et écart type

\begin{tabular}{lcccccccccc}
\hline Variables & V1 & V2 & V3 & V4 & V5 & V6 & V7 & V8 & $\begin{array}{r}\text { Moyenne Écart } \\
\text { type }\end{array}$ \\
\hline $\begin{array}{l}\text { Croissance } \\
\text { CA }\end{array}$ & 1,00 & & & & & & & & 2,75 & 0,56 \\
\hline $\begin{array}{l}\text { Domaines } \\
\text { d'activité }\end{array}$ & 0,03 & 1,00 & & & & & & 2,52 & 0,67 \\
\hline $\begin{array}{l}\text { CA avec } \\
\text { anciens clients }\end{array}$ & $-0,16$ & $0,23^{*}$ & 1,00 & & & & & & 2,57 & 0,63 \\
\hline $\begin{array}{l}\text { Nombre } \\
\text { de clients }\end{array}$ & $-0,08$ & 0,16 & $0,45^{* * *}$ & 1,00 & & & & & 2,08 & 0,39 \\
\hline $\begin{array}{l}\text { Nombre } \\
\text { de produits }\end{array}$ & $0,40^{* * *}$ & 0,00 & 0,03 & $-0,13$ & 1,00 & & & & 2,49 & 0,63 \\
\hline $\begin{array}{l}\text { Qualité } \\
\text { des produits }\end{array}$ & $0,29^{* *}$ & $0,34^{* * *}$ & 0,16 & $0,39^{* * *}$ & 0,14 & 1,00 & & 2,49 & 0,50 \\
\hline $\begin{array}{l}\text { Qualité } \\
\text { des services }\end{array}$ & 0,03 & 0,17 & $0,36^{* * *}$ & 0,04 & $0,020^{* *}$ & 0,13 & 1,00 & 2,47 & 0,50 \\
\hline \begin{tabular}{l} 
Prix \\
\hline
\end{tabular} & $0,26^{*}$ & 0,13 & $-0,22^{*}$ & $-0,21$ & $0,31^{* *}$ & $-0,18$ & 0,01 & 1,00 & 1,94 & 0,59 \\
\hline
\end{tabular}

$\mathrm{N}=83,{ }^{*} \mathrm{p}<0,05 ; *{ }^{*} \mathrm{p}<0,01 ; * * * \mathrm{p}<0,001$. 


\section{Hypothèse 1}

L'analyse de variance multivariée (MANOVA: Wilk $F=1,36$, n.s.) montre que l'origine de la transmission ne permet pas de dire que les changements stratégiques apportés par les repreneurs internes sont différents des changements stratégiques apportés par les repreneurs externes. Les tests effectués à partir des analyses univariées (ANOVA) aboutissent au même résultat (tableau 2).

TABLEAU 2

Valeur des $F$ en fonction du type de transmission, de la taille et de la situation financière des entreprises

\begin{tabular}{lccc}
\hline Variables & $\begin{array}{c}\text { Transmissions internes / } \\
\text { Transmissions externes } \\
\text { Valeur de F }\end{array}$ & $\begin{array}{c}\text { Taille < 200/ } \\
\text { Taille }>\mathbf{2 0 0} \\
\text { Valeur de F }\end{array}$ & $\begin{array}{c}\text { Bénéficiaire / } \\
\text { Non bénéficiaire } \\
\text { avant transmission } \\
\text { Valeur de F }\end{array}$ \\
\hline Croissance CA & 0,057 & 0,07 & 0,29 \\
CA avec anciens clients & 0,02 & 0,72 & 0,04 \\
Nombre de clients & 2,13 & 0,04 & 1,53 \\
Secteurs d'activités & 0,74 & 0,72 & 0,17 \\
Nombre de produits & 0,47 & 0,34 & 0,03 \\
Qualité des produits & 0,13 & 0,02 & $3,85^{*}$ \\
Qualité des services & 2,00 & 0,03 & 0,052 \\
Prix & 0,48 & $4,34^{* *}$ & 2,66 \\
\hline
\end{tabular}

${ }^{*} \mathrm{p}<0,10 ; * * \mathrm{p}<0,05$.

Pour vérifier si la taille des entreprises et leur situation financière avant la transmission ont un effet sur les changements stratégiques, des analyses de variance ont été réalisées. La comparaison entre les entreprises ayant un effectif inférieur à 200 salariés et celles ayant un effectif supérieur à 200 salariés, effectuée à partir de l'analyse multivariée (Wilk $F=1,12$, n.s.), n'est pas significative. Les tests effectués à partir des analyses univariées aboutissent au même résultat excepté pour la variable « prix des produits » (tableau 2). Pour la situation financière au moment de la transmission, l'analyse de variance multivariée montre qu'il n'y a pas de différence significative entre les entreprises bénéficiaires et celles qui sont déficitaires au moment du rachat (Wilk $F=1,17$, n.s.). Les tests effectués individuellement aboutissent au même résultat à l'exception de la variable «qualité » des produits (tableau 2).

En conséquence, les résultats nous poussent à rejeter notre première hypothèse. Par ailleurs, il semble que ni la taille de l'entreprise ni sa situation financière avant le rachat ne soient des variables permettant de différencier les changements stratégiques opérés par les entreprises dont la direction a changé de mains. 


\section{Hypothèse 2}

Les résultats de l'analyse typologique montrent que cinq groupes ont été formés à partir des huit variables stratégiques (tableau 3). Pour toutes les variables, la valeur de $\mathrm{F}$ est significative au seuil de $5 \%$; autrement dit, chaque groupe est différent.

TABleau 3

Analyse typologique des variables stratégiques

\begin{tabular}{|c|c|c|c|c|c|c|}
\hline Variables & $\begin{array}{c}\text { Groupe I } \\
n=27\end{array}$ & $\begin{array}{c}\text { Groupe II } \\
n=21\end{array}$ & $\begin{array}{c}\text { Groupe III } \\
n=20\end{array}$ & $\begin{array}{c}\text { Groupe IV } \\
n=10\end{array}$ & $\begin{array}{c}\text { Groupe } \mathrm{V} \\
n=5\end{array}$ & $\begin{array}{l}\text { ANOVA } \\
\text { Valeur F }\end{array}$ \\
\hline $\begin{array}{l}\text { Croissance } \\
\text { CA }\end{array}$ & 3,0 & 2,28 & 3,0 & 3,0 & 1,8 & $18,77^{*}$ \\
\hline $\begin{array}{l}\text { CA avec } \\
\text { anciens clients }\end{array}$ & 1,88 & 3,0 & 2,9 & 3,0 & 1,4 & $54,58 *$ \\
\hline $\begin{array}{l}\text { Nombre } \\
\text { de clients }\end{array}$ & 2,48 & 2,95 & 2,85 & 2,0 & 1,4 & $16,70 *$ \\
\hline $\begin{array}{l}\text { Secteurs } \\
\text { d'activité }\end{array}$ & 2,0 & 2,14 & 2,3 & 2,0 & 1,60 & $4,77^{*}$ \\
\hline $\begin{array}{l}\text { Nombre } \\
\text { de produits }\end{array}$ & 2,70 & 2,0 & 2,95 & 2,8 & 1,60 & $17,16^{*}$ \\
\hline $\begin{array}{l}\text { Qualité } \\
\text { des produits }\end{array}$ & 2,48 & 2,47 & 2,9 & 2,0 & 2,0 & $9,86^{*}$ \\
\hline $\begin{array}{l}\text { Qualité } \\
\text { des services }\end{array}$ & 2,11 & 2,38 & 2,95 & 2,70 & 2,40 & $14,47 *$ \\
\hline Prix & 2,03 & 1,52 & 2,2 & 1,9 & 2,2 & $4,72 *$ \\
\hline $\begin{array}{l}\text { Perception de } \\
\text { l'environnement }\end{array}$ & $\begin{array}{l}\text { - compétition } \\
\text { plus intensive } \\
\text { - pas de } \\
\text { possibilité de } \\
\text { lutter sur } \\
\text { le marché } \\
\text { actuel }\end{array}$ & $\begin{array}{l}\text { - compétition } \\
\text { forte } \\
\text { - environne- } \\
\text { ment instable } \\
\text { - volonté de } \\
\text { rester sur } \\
\text { le marché } \\
\text { actuel }\end{array}$ & $\begin{array}{l}\text { - compétition } \\
\text { plus intense } \\
\text { - volonté de } \\
\text { rester sur le } \\
\text { marché } \\
\text { actuel } \\
\text { - volonté de } \\
\text { développer } \\
\text { d'autres } \\
\text { produits }\end{array}$ & $\begin{array}{l}\text { - environ- } \\
\text { nement } \\
\text { favorable } \\
\text { - volonté de } \\
\text { satisfaire } \\
\text { les clients } \\
\text { actuels }\end{array}$ & $\begin{array}{l}\text { - manque de } \\
\text { ressources } \\
\text { - obligation de } \\
\text { recentrer sur } \\
\text { activités } \\
\text { profitables } \\
\text { - repenser } \\
\text { l'organisation } \\
\text { différemment }\end{array}$ & \\
\hline
\end{tabular}

$* \mathrm{p}<0,001$

D’après les résultats présentés au tableau 3, les groupes peuvent être décrits de la manière suivante :

\section{Groupe I: Stratégie orientée vers un «nouveau positionnement »}

Les entreprises ayant opté pour une stratégie orientée vers un nouveau positionnement ont été repérées par une augmentation du taux de croissance du chiffre d'affaires, du nombre de produits, du nombre de clients ainsi que de la qualité des produits. 
Mais le nombre de domaines d'activité, le prix des produits, le chiffre d'affaires réalisé avec les anciens clients et la qualité des services restent identiques à ceux du prédécesseur.

D'après les questions ouvertes, on peut supposer que les entreprises de ce groupe cherchent à étendre le champ de leur marché actuel en offrant de nouveaux produits et en cherchant de nouveaux clients. Les dirigeants interrogés s'expliquent en évoquant l'entrée de nouvelles entreprises qui empiètent sur leur territoire et qui rendent la compétition plus intense. Une de leurs réponses consiste alors à développer de nouveaux produits pour une nouvelle clientèle.

\section{Groupe II : Stratégie orientée vers un « maintien »}

La stratégie orientée vers un développement se caractérise par une augmentation du chiffre d'affaires sur les anciens clients, du nombre de clients, de la qualité des produits et des services. Elle est aussi le reflet d'une diminution du prix des produits et du maintien à l'identique du taux de croissance du chiffre d'affaires, des domaines d'activité et du nombre de produits.

Les questions ouvertes font ressortir le fait que les entreprises appartenant à ce groupe sont localisées dans un marché où la concurrence est forte et où l'environnement est décrit comme étant intense et instable. Selon les dirigeants, pour se maintenir dans leur secteur d'activité, leurs entreprises doivent réduire les prix des produits tout en améliorant leur qualité en permanence.

\section{Groupe III : Stratégie orientée vers un « développement externe »}

Les entreprises de ce groupe sont caractérisées par une augmentation du taux de croissance du chiffre d'affaires, du chiffre d'affaires réalisé avec les anciens clients, du nombre de clients, du nombre de produits ainsi que de la qualité des produits et de la qualité des services. Par ailleurs, le nombre des domaines d'activité et le prix des produits comparés à ceux du prédécesseur sont sensiblement identiques.

Il ressort des questions ouvertes que les orientations stratégiques prises sont avant tout une réaction à une compétition intense et que, même s'il existe une volonté de rester sur son marché actuel, dans la mesure où il est porteur, il existe aussi une volonté de développer de nouveaux produits.

\section{Groupe IV : Stratégie orientée vers un « développement interne »}

Les entreprises dont la stratégie est orientée vers un développement interne sont plutôt caractérisées par une augmentation du taux de croissance du chiffre d'affaires, du chiffre d'affaires réalisé avec les anciens clients, du nombre de produits et de la 
qualité des services. De même, il a été constaté que le nombre de clients, le nombre de domaines d'activité, la qualité des produits ainsi que le prix des produits étaient identiques à ceux du prédécesseur.

Les dirigeants perçoivent l'environnement comme étant favorable puisque les besoins des clients actuels sont satisfaits et que le système relationnel établi avec leurs anciens clients est maintenu. Pour eux, il n'est pas nécessaire d'apporter un changement profond pour attirer de nouveaux clients.

\section{Groupe V : Stratégie orientée vers le « retrait »}

Les entreprises qui semblent prendre une stratégie orientée vers le retrait sont caractérisées par une diminution du nombre de domaines d'activité, du nombre de produits, du nombre de clients et du chiffre d'affaires réalisé avec les anciens clients. Dans le même temps, les dirigeants choisissent d'augmenter les prix et la qualité des services, mais la qualité des produits reste équivalente, de même que la croissance du chiffre d'affaires par rapport au prédécesseur.

À l'examen des questionnaires, nous relevons que les nouveaux dirigeants justifient leur choix en soulignant le caractère de rationalisation favorisant une logique de profit plutôt qu'une logique de volume. Il s'agit pour eux de se repositionner avec une gamme de produits moins étendue dans un circuit de distribution plus absorbant, c'est-à-dire de passer d'un circuit de distribution de détail à un circuit de distribution de grossiste.

De plus, une analyse de variance multivariée (MANOVA) réalisée sur l'ensemble des groupes permet d'aboutir aux mêmes conclusions (Wilk $F=17,17, p<0,001$ ). Pour chaque groupe, la perception de l'environnement est différente et les orientations stratégiques sont déterminées par la perception du contexte environnemental dans lequel l'entreprise évolue.

En conséquence, les résultats nous poussent à accepter la seconde hypothèse. En complément de ces résultats, des analyses du khi carré ont été réalisées. Voici ce qu'il en ressort :

- la répartition des transmissions internes et des transmissions externes s'effectue de façon aléatoire sur l'ensemble de l'échantillon (tableau 4);

- la répartition entre les entreprises ayant un effectif inférieur à 200 salariés et celles ayant un effectif compris entre 200 et 500 salariés s'effectue de façon aléatoire sur l'ensemble de l'échantillon (tableau 5);

- la répartition des entreprises bénéficiaires et celles qui sont déficitaires s'effectue de façon aléatoire sur l'ensemble de l'échantillon (tableau 6). 
Aussi, ces résultats nous obligent à conclure que ni l'origine de la transmission (transmission interne / transmission externe), ni la taille des entreprises (moins de 200 salariés / plus de 200 salariés), ni la situation financière (bénéficiaire / déficitaire) ne peuvent différencier les groupes mis en évidence par l'analyse typologique.

\section{TABLEAU 4}

Répartition des transmissions internes et externes

\begin{tabular}{lcccccc}
\hline $\begin{array}{l}\text { Type de } \\
\text { transmission }\end{array}$ & $\begin{array}{c}\text { Échantillon } \\
\text { total } \\
n=83\end{array}$ & $\begin{array}{c}\text { Groupe I } \\
n=27\end{array}$ & $\begin{array}{c}\text { Groupe II } \\
n=21\end{array}$ & $\begin{array}{c}\text { Groupe III } \\
n=20\end{array}$ & $\begin{array}{c}\text { Groupe IV } \\
n=10\end{array}$ & $\begin{array}{c}\text { Groupe V } \\
n=5\end{array}$ \\
\hline $\begin{array}{l}\text { Transmissions } \\
\text { internes }\end{array}$ & 53 & 18 & 11 & 15 & 6 & 3 \\
\hline $\begin{array}{l}\text { Transmissions } \\
\text { externes }\end{array}$ & 30 & 9 & 10 & 5 & 4 & 2 \\
\hline$\chi^{2}=2,46$ (ddl =4, n.s.) & & & & &
\end{tabular}

TABLEAU 5

Distribution en fonction de la taille

\begin{tabular}{lcccccc}
\hline $\begin{array}{l}\text { Type de } \\
\text { transmission }\end{array}$ & $\begin{array}{c}\text { Échantillon } \\
\text { total } \\
n=83\end{array}$ & $\begin{array}{c}\text { Groupe I } \\
n=27\end{array}$ & $\begin{array}{c}\text { Groupe II } \\
n=21\end{array}$ & $\begin{array}{c}\text { Groupe III } \\
n=20\end{array}$ & $\begin{array}{c}\text { Groupe IV } \\
n=10\end{array}$ & $\begin{array}{c}\text { Groupe V } \\
n=5\end{array}$ \\
\hline$<200$ salariés & 54 & 15 & 11 & 16 & 7 & 5 \\
\hline 200 salairés & 29 & 12 & 10 & 4 & 3 & 0 \\
\hline
\end{tabular}

$\chi^{2}=7,31(\mathrm{df}=4$, n.s. $)$

TABLEAU 6

Distribution en fonction de la situation financière

\begin{tabular}{lcccccc}
\hline $\begin{array}{l}\text { Type de } \\
\text { transmission }\end{array}$ & $\begin{array}{c}\text { Échantillon } \\
\text { total } \\
n=83\end{array}$ & $\begin{array}{c}\text { Groupe I } \\
n=27\end{array}$ & $\begin{array}{c}\text { Groupe II } \\
n=21\end{array}$ & $\begin{array}{c}\text { Groupe III } \\
n=20\end{array}$ & $\begin{array}{c}\text { Groupe IV } \\
n=10\end{array}$ & $\begin{array}{c}\text { Groupe V } \\
n=5\end{array}$ \\
& & & & & \\
\hline
\end{tabular}

Bénéficiaire

au moment

du rachat

76

25

20

18

8

5

Déficitaire

au moment

\begin{tabular}{lllllll} 
du rachat & 7 & 2 & 1 & 2 & 2 & 0 \\
\hline$\chi^{2}=2,65(\mathrm{df}=4$, n.s. $)$ & &
\end{tabular}




\section{Discussion des résultats et conclusion}

Cette recherche s'est attachée à mieux comprendre les liens qui existaient entre repreneurs internes et repreneurs externes, ainsi que les changements d'orientations stratégiques qui suivent la transmission. Elle a mis en évidence que l'origine de la transmission (transmission interne ou transmission externe) ne permet pas de prédire les différences dans les changements et les orientations stratégiques adoptées par les nouveaux dirigeants. Il a été constaté également que ni la taille, ni la situation financière de l'entreprise avant le rachat ne permettent de prédire de différences significatives dans les changements stratégiques.

Par ailleurs, il ressort de ce travail que les orientations stratégiques mises en place après la transmission du dirigeant pouvaient prendre différentes formes. Cinq groupes ont été définis à partir, d'une part, des variations constatées des variables stratégiques et, d'autre part, à partir des perceptions que se font les dirigeants de l'environnement dans lequel évolue leur entreprise. S'il est admis que les orientations stratégiques sont déterminées par la perception qu'a le nouveau dirigeant de l'environnement, il faut souligner que la probabilité de voir apparaître de nouvelles orientations stratégiques s'accroît, non seulement avec l'occurrence d'un changement de conditions environnementales, mais aussi parce que le nouveau dirigeant perçoit ce changement. La perception de l'environnement est un événement crucial pour l'entreprise dans la mesure où il est le vecteur initial des actions managériales qui vont être menées, et que ces actions managériales sont davantage liées aux réalités perceptuelles qu'à des réalités objectives (Meyer, 1982).

Pour autant, s'il a été montré que les changements stratégiques et les nouvelles orientations stratégiques ne peuvent être différenciés en fonction du type de transmission de dirigeant, d'autres pistes doivent être explorées afin de rendre compte de la complexité organisationnelle dans laquelle évolue l'entreprise.

1. L'une de ces pistes serait l'étude du système perceptif des dirigeants dans la mesure où il a été démontré qu'il influe de manière déterminante sur les choix stratégiques des entreprises. C'est donc en intégrant dans les recherches à venir des éléments permettant de saisir la manière dont le dirigeant appréhende l'environnement que l'on réussira à mieux comprendre les stratégies élaborées par les nouveaux dirigeants de PME, et surtout les changements et la diversité des orientations stratégiques élaborées par les repreneurs.

2. Une autre de ces pistes consisterait à étudier la structure de propriété des entreprises qui ont été transmises. À l'heure actuelle, seule une distinction binaire a été effectuée entre les repreneurs internes et les repreneurs externes, mais il serait tout aussi légitime d'intégrer une distinction dans le type de transmission à partir des différentes structures de propriété. En 
effet, le mode de gouvernement qui anime les entreprises pourrait ainsi expliquer certains des choix stratégiques posés par les PME lors de leur transmission.

\section{Bibliographie}

ALDRICH, H. (1979), Organizations and Environment, Englewood Cliffs, New Jersey, Prentice-Hall.

BARCELO, R. (1988), «Transmission héréditaire et système de production : le cas de la Soule », Sociologie du travail, vol. 3, p. 443-460.

BROKAW, L. (1992), «Why family are the best », INC, vol. 14, mars, p. 72-81.

BuChele, R.B. (1965), Business Policy in Growing Firms, San Francisco, Chandler.

CEPME (1990), «La transmission des PME», dans Les études du CEPME, juin, 22 p.

CHAGANTI, R. et J.A. SCHNEER (1994), «A study of the impact of owner's mode of entry on venture performance and management patterns », Journal of Business Venturing, vol. 9 , p. 243-260.

CHILD, J. (1972), «Organizational structure, environments and performance: the role of strategic choice », Sociology, vol. 6, p. 1-22.

ChURCHILl, N.C. et K.J. HATTEN (1987), «Non-market based transfers of wealth and power : a research framework for family businesses », American Journal of Small Business, vol. 11, p. 51-64.

COOPER, A.C. et W.C. DunkelBerg (1986), «Entrepreneurship and paths to business ownership », Strategic Management Journal, vol. 7, p. 53-68.

DAFT, R.L., J. SORMUNEN et D. PARKS (1988), «Chief executive scanning, environmental characteristics, and company performance : an empirical study », Strategic Management Journal, vol. 9, p. 123-139.

D’Amboise, G., et M. Muldowney (1988), « Management theory for small business : attempts and requirements », Academy of Management, vol. 13, p. 226-240.

DodGe, H.R., S. FULLERTON et J.E. RoBbINS (1994), « Stage of the organization life cycle and competition as mediators of problem perception for small business », Strategic Management Journal, vol. 15, p. 121-134.

DODGSON, M. et R. ROTHWELL (1989), « Technology strategy in small and medium-sized firms », dans M. Dodgson (dir.), Technology Strategy and the Firm, Harlow, Longman.

GARACH, J.A. et J.B. GANITSKY (1995), «Successful transmission in family business », Family Business Review, vol. 8, p. 131-152.

GARTNER, W.B. (1985), «Conceptual framework for describing phenomenon of new venture creation », Academy of Management Review, vol. 10, p. 696-706.

GreEnFIELD, S.M. et A. STRICKON (1981), «A new paradigm for the study of entrepreneurship and social change », Economic Development and Cultural Change, vol. 29 , p. 467-499. 
GREINER, L.E. (1972), «Evolution and revolution as organizations grow », Harvard Business Review, vol. 50, p. 37-46.

HAMBRICK, D.C. (1984), « Taxonomic approaches to studying strategy : some conceptual and methodological issues », Journal of Management, vol. 26, p. 193-206.

HANDLER, W.C. (1991), « Key interpersonal relationships of next generation family members in family firms », Journal of Small Business Management, vol. 29, p. 21-32.

HANDLER, W.C. (1994), «Transmission in family business : a review of the research", Family Business Review, vol. 7, p. 132-157.

HiRIgoyen, G. (1987), « La transmission des PME familiales : constat et suggestions », La Revue du Financier, ${ }^{0}$ 55, juillet-août, p. 37-48.

INSEE (1997), «Les repreneurs d'entreprises », $n^{\circ} 509$, février.

JICK, T. (1979), «Mixing qualitative and quantitative methods : triangulation in action », Administrative Science Quarterly, vol. 24, p. 602-611.

JuLIEN, P.A. (1994), Les PME : Bilan et perspectives, Cap-Rouge, Presses Inter Universitaires ; Paris, Economica, 437 p.

KETS DE VRIES, M.F.R. (1985), « The dark side of entrepreneurship », Harvard Business Review, vol. 63, p. 160-167.

LANSBERG, I. et J.H. ASTRACHAN (1994), « Influence of family relationships on transmission planning and training: the importance of mediating factors », Family Business Review, vol. 7, p. 39-59.

LEHMANN, P.J. (1993), «Le financement de la transmission des PME », Revue française de gestion, $\mathrm{n}^{\circ}$ 95, septembre-octobre, p. 116-121.

MERZ, G.R. et M.H. SAUBER (1995), «Profiles of managerial activities in small firms », Strategic Management Journal, vol. 16, p. 551-564.

MEYER, A.D. (1982), «Adaptation to environmental jolts », Administrative Science Quarterly, vol. 27, p. 515-537.

NIEMANN, H. (1991), «Buying a business : the rest of the story », INC, vol. 13, octobre, p. $38-47$.

ORPEN, C. (1994), « Strategic planning, scanning activities and the financial performance of small firms », Journal of Strategic Change, vol. 3, p. 45-55.

Perreault, Y.G. (1994), L'entreprise familiale, Montréal, Les Éditions Transcontinental, Fondation de l'entrepreneurship.

PORTER, M.E. (1985), L'avantage concurrentiel, Paris, InterÉditions.

ROBINSON, R.B. et A. PEARCE (1984), « Research thrusts in small firm strategic planning », Academy of Management Review, vol. 9, p. 128-137.

SEXTON, D.L. et P.M. VAN AUKEN (1982), « Prevalence of strategic planning in the small business », Journal of Small Business Management, vol. 20, p. 20-26.

SILVESTRE, H. et R. GOUJET (1996), «Lisibilité de l'environnement, management stratégique : éléments de recherche sur les PMI », Revue internationale PME, vol. 9, $\mathrm{n}^{\circ}$ 1, p. 61-78. 
SteVEnSON, H.H., M.J. RoBerTS et H.I. GRousbeCK (1985), New Business Ventures and the Entrepreneur, Homewood, Illinois, Richard Irwin.

STONER, C.R. (1982), « Planning in small manufacturing firms : a survey », Journal of Small Business Management, vol. 31, p. 34-41.

THIÉTART, R.A. (1987), La stratégie d'entreprise, Paris, McGraw-Hill.

WARD, J.L. (1987), Keeping the Family Business Healthy, San Francisco, Jossey Bass.

WeIcK, K.E. (1969), The Social Psychological of Organizing, Reading, Mass., AddisonWesley.

WEINSHAL, T.D. et L. VICKERY (1987), «Entrepreneurs : a balanced view of their role in innovation and growth », European Management Journal, vol. 5, n 4, p. 257-267.

WiLlARD, G.E. et D.A. KRUEGER (1992), «In order to grow, must the founder go : a comparison of performance between founder and non-founder managed high-growth manufacturing firms », Journal of Business Venturing, vol. 7, p. 181-194. 